\title{
EDITORIAL
}

\section{In View of Today's Realities: What should any Work with the Plant World Actually Gain us?}

\author{
Sener Akinci* \\ Marmara University, Turkey
}

ARTICLE INFO

Article history

Received: 4 November 2021

Accepted: 5 November 2021

Published Online: 8 November 2021
Studies on plants are important evidence not only for their diversity and richness in the world, but also for revealing the relationship between the environment and the organisms. How important are the concepts of climate crisis and global warming? What is the number of people in the world, other than scientists, who are aware of the seriousness of the problem? Do we have any information about the number of people who have knowledge about what can be done? Do we want to experience the carbon richness of millions of years ago (Carboniferous) again? Let's not forget that this reality, which seems to be an advantageous situation at first glance, has actually been moved to a platform where today's leaders discuss the effects of climate balance. We need more and more plants all over the planet. With the increase in the human population, it is time to change our prejudices about "uncertain or suspicious" plants to be used. Could some toxic metabolites be usable or even edible by appropriate treatments? Can countries that are lucky in terms of endemic species make better use of these reserves? Plant biochemistry studies should be encouraged in this respect.
In addition, there is evidence that the consumption of a large number of products that we use as food causes health problems that we do not know yet, but which can increase greatly with daily use and even be fatal when contaminated by pathogenic organisms.

What are the ways to safely store our food without using any additives? Food safety concerns many topics from the quality of herbal products to their shelf life. For example, if we can produce coffee, which is one of the most important economic plants of the world economy, against the climate crisis and moreover, if we can increase the amount of production, how can we ensure long-term protection of these coffee fruits/seeds?

Will the multitude of countries whose economies depend entirely on the cultivation and marketing of plantation products such as bananas (Musa Spp), coffee (Coffea arabica L.) and cacao (Theobroma cacao L.) overcome their biggest obstacle, the dual problems? There will undoubtedly be a great need for all kinds of scientific research and efforts to support this issue. Plant researches will give important clues about

*Corresponding Author:

Sener Akinci,

Marmara University, Turkey;

Email: akincisener23@gmail.com 
maintaining the harmony of human and nature with good study topics selections.

Any study on botany will reinforce our knowledge in persuading us to reveal their importance in our lives with more and more sharp lines, based on the fact that the first human probably was the first food of a plant. Scientific publications are unique resources that will assume their responsibility in this regard.

Here in this issue of the journal, we present evidence that the more intensive plant researches are done, the more they will contribute to the maintenance of human-nature relations in an ecological balance. 